\title{
TID selection and research of its characteristics on ionograms
}

\author{
Akchurin A.D., Bochkarev V.V., Ildiryakov V.R., Usupov K.M. \\ Kazan (Volga Region) Federal University (KFU); Russia Federation, Kazan, 420008, Kremlevskaya st., 18, \\ tel:+7(843)2337177, e-mail: adel.akchurin@ksu.ru
}

\begin{abstract}
The various manifestations of TID in ionograms received with minute periodicity by Kazan $\left(55^{\circ} \mathrm{N}, 48^{\circ} \mathrm{E}\right)$ ionosonde during winter time are considered. Besides a U-shaped TID trace traditionally observable in ionogram and corresponding intensive inhomogeneities are revealed focusing TID which corresponding low-intensity inhomogeneities. The focusing TID detection became possible owing to a gain choice which excludes restriction of amplitude of the reflected signal. Use of a three-dimensional array of amplitudes of F-region echoes has allowed to determine a basic parameters of TID such as periodicity and descent time.
\end{abstract}

\section{Introduction}

The basic method of recording of travelling ionospheric disturbances (TID) is radio sounding which has made their basic classification of time periods: large-, medium- and small-scale (LS, MS and SS respectively). The first group is made of TID with periods more than $1 \mathrm{~h}$, the second group - with periods in a range of 20-60 min and the third - 1-10 min [1-3]. With sixties till now for analysis TID the new frequencies and sounding methods were stage by stage involved and together with more sensitive processing techniques they have formed a basis of alternative TID classification of the sizes. It has appeared not such simple as three-componental classifications of time periods that it seems to be related both with the various nature of formation of irregularities and with complexity and ambiguity of performance of inverse problems. So according to one of the up-to-date and fullest classifications [1] "kernel" of the first group of LS TID is made by disturbances with the horizontal sizes (wave length) over $1000 \mathrm{~km}$, the second group of MS TID - in a range from 50 to $300 \mathrm{~km}$ and the third SS TID - in a range from 0.1 to $1 \mathrm{~km}$. For "gap" closing between SM and SS TID in [1] are entered TID of the intermediate scale (IS) with the sizes $1-30 \mathrm{~km}$.

Separation of MS and IS irregularities is entered because of difference their forms in the various methods of radio sounding specifying in various physical mechanisms of formation. The least quantity of the information is had about the physics of formation of IS irregularities (also as well as about SS). The role of the direct originator in creation of F-spread and scintillation of radio signals is allocated by it in the literature. The information about MS irregularities more versatile so they are observed by set of methods in a role of the generator of an original interference field of perturbations [1, 4]. In particular, MS TID, observed in the HFrange, have various forms depending on intensity of irregularities of perturbation $\delta=\Delta \mathrm{N}_{\mathrm{e}} / \mathrm{N}_{\mathrm{e}}^{\text {backgr }}$. At vertical sounding MS TID depending on their intensity $\delta$ can are observed as $[5,6,8]: 1)($ at $\delta>10 \%$ ) separate in U- or nose- shaped traces detached from the parent layer within which they were formed; 2 ) (at $1 \%<\delta<10 \%$ ) variations of virtual heights of reflection $(\Delta h>20 \mathrm{~km})$ on a part of a trace of an ionospheric layer with possible occurrence of an kink; 3) (at $\delta<1 \%$ ) variations of virtual heights of reflection $(\Delta h<20 \mathrm{~km})$ generally observed on an interference pattern (formed by modes with various polarization). Boundary values $\delta$ are approximate as the disturbance intense $(\delta)$ is detemined extremely roughly at vertical sounding [6]. Weak intensive irregularities were often observed in records of Doppler-shift signal, variations of a group path of various rays (propagation modes), it detected at oblique sounding by complicated coherent (interference) techniques using of spatial and frequency diversities [1].

The MS and IS heterogeneities are the least studied which often are weak intensive $(\delta<10 \%)$ and poorly noticeable on ionograms. To their recording we apply a method of determination of height of reflection with improved precision [7], allowing to obtain height variations with average accuracy of $200 \mathrm{~m}$ for not diffuse reflections. Except the these technique in this paper we give the other ones for the study of MS TID based on a more frequent rate of sounding without overamplification (saturation of output receiver stage) and on mapping of the sounding information in the form of convenient for TID separation with the minimum amount of manual labor.

\section{Data processing results}

For reliable MS TID detection was performed two months (January, February, 2011.) vertical sounding of the ionosphere with minute periodicity of ionogram registrations. The dynamic range of the signal reflected 
from an ionosphere is enough great, therefore often the receiver operates with an gain level exceeding necessary for recording usual (not diffused and not strong absorption) reflections. By such level there is a possibility to observe weak diffuse signals, and traces of ionospheric layers look brighter and thicker, but the form of normally reflected signals is deformed by overamplification. Normal operation of our method for height determining with high accuracy requires the registration of the exact form of the reflected signal, therefore distinctive feature of the probe was the work of the receiver with such a gain, which does not distort the shape of the large amplitude of the reflected signal.

Up to half of TID cases in daytime ionograms were the additional nose-shaped traces travelled from critical frequency of F-layer to critical frequency of E-layer with average time of descent of $\sim 10 \mathrm{~min}$. Characteristic feature such TIDs are the raised amplitude of a nose tip (see Fig. 1) which is well visible in a colored scale amplitudes. A series of ionograms with 1 min steps (time increase from left to right and from top to down) is presented in fig. 1 where the raised amplitude is highlighted in black color and, besides, in an amplitude-frequency characteristic (AFC) of F-trace located under everyone ionograms it is marked by an arrow.
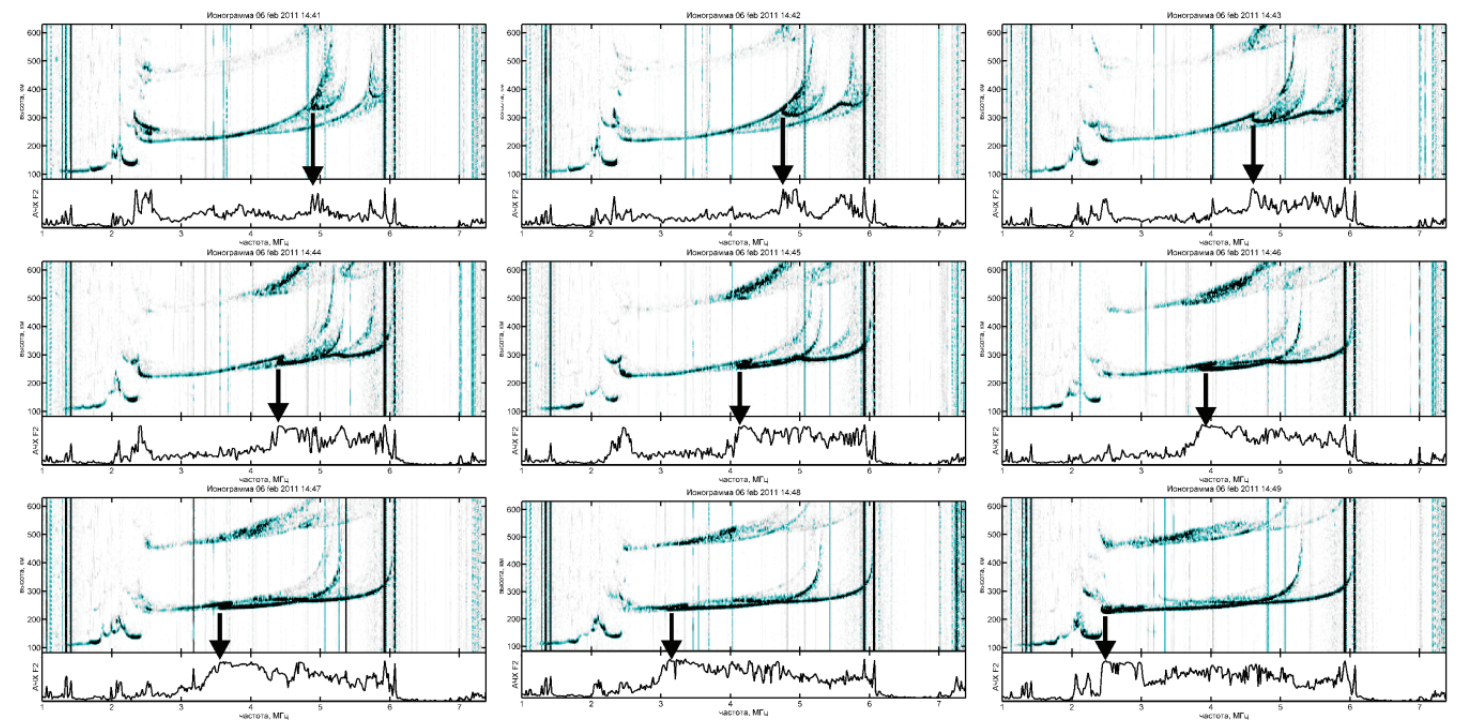

Fig. 1. A series of ionograms showing advance of nose-shaped TID along a F-layer trace. The raised amplitude of tip of nose-shaped TID is highlighted in black color and indicated by an arrow in AFC plot

The rest of cases of travelled irregularities have made small intensity TID which could not change considerably reflection height $(\Delta h<5 \mathrm{~km})$ to form a kink in F-layer trace. Nevertheless TID occurrence of this kind is visible not only by means of our method of virtual height estimation with improved precision [7], but also in AFC of F-trace as segment with the raised amplitude (see fig. 2), tentatively called us as a focusing of a trace segment. Such trace focusings and also nose tips of intensive TID move from right to left, i.e. downwards from critical frequency of F-layer. At approach to critical frequency of F1-layer (or E-layer) the location of focusing is often transmuted into an S-shaped curl on a trace of F1-layer. The S-shaped curl also has the raised amplitude and moves to critical frequency of E-layer. Time of descent such focusing TID is approximately equal to tidal time of nose-shaped TID.

The raised amplitude of tip of nose-shaped intensive TID and of focusing TID creates prerequisite of the automated analysis of occurrence frequency of TID and measurement of descent time and occurrence frequency. For this purpose the maxima of the signals reflected $>200 \mathrm{~km}$ (one maximum on each frequency) were selected in each ionogram and united in the AFC of F-layer trace. Before formation of AFC of F-layer traces the wavelet-filtration was applied for effectively deleting noise with ionograms. The diurnal set of such AFC assembled in a three-dimensional array was processing for search of "descent lines". The fragment of such diurnal three-dimensional array of AFC of F-layer echoes, briefly named by us an ATF-plot of layer F, is shown in fig. 3. Upper border of ATF corresponds to fxF2 variations, and bottom - foE. Dark streaks on the ATF bottom part concerning reflections of an o-mode correspond to the descent lines formed by moving sections of TID traces with raised amplitudes. The analysis of such streaks has shown that focusing TIDs appear most often (approx. 30 times per day, with average time of a descent of $7 \mathrm{~min}$ ). The nose-shaped TIDs occur on the average 7 times per day with average time of a descent 10 minutes. The S-shaped curl on a trace of F1-layer is observed about 5 times per day with average time of a life 5 minutes. 

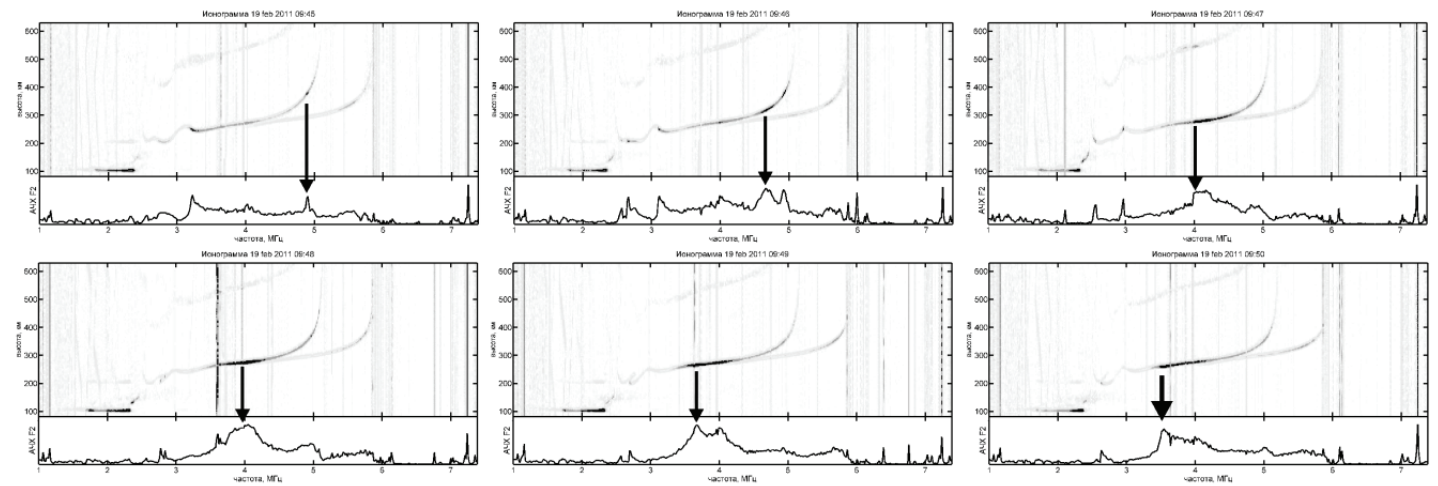

Fig. 2. A series of ionograms, showing to feature of advance TID, in the form of site of AFC of F-layer strengthened on amplitude. The site of the raised amplitude is highlighted in black color and indicated by an arrow in AFC plot

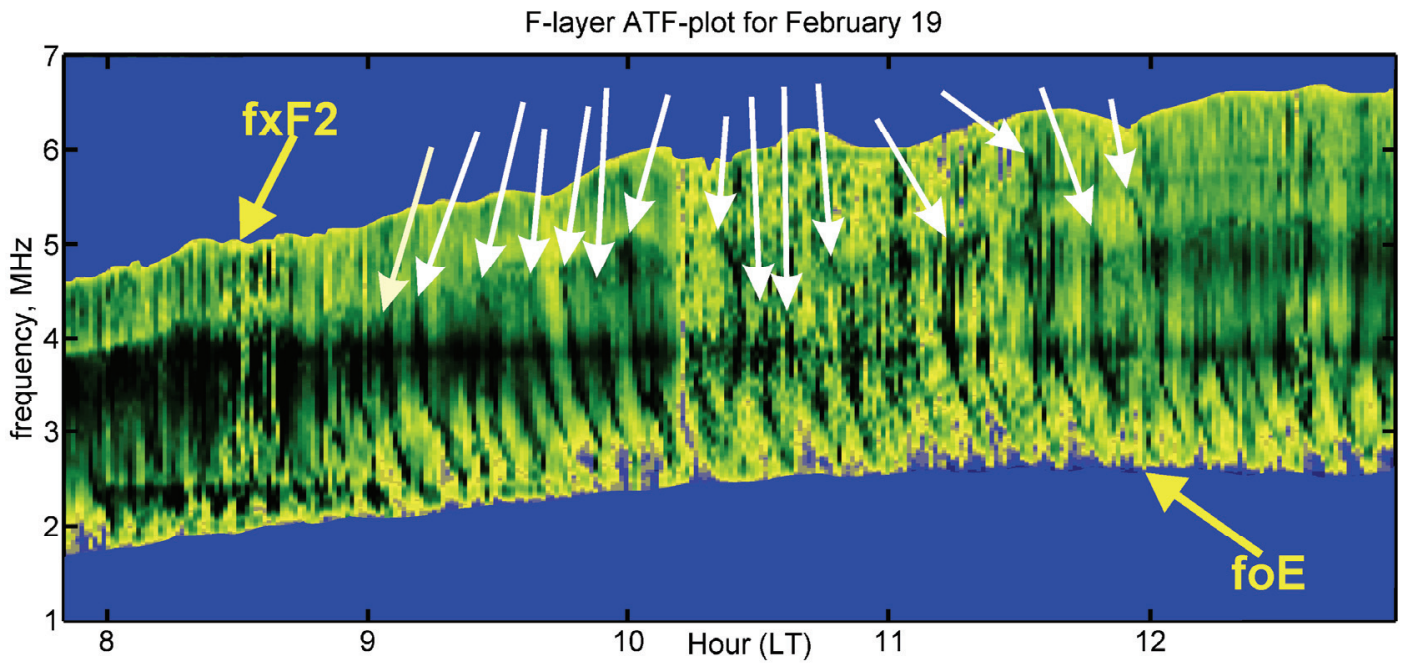

Fig. 3. A fragment of daily three-dimensional array of AFC of reflections from F-layer (a fragment ATF-plot). Vertical streaks of black color correspond to lines of descent of TID various intensity

\section{Conclusion}

The speeded up measurements of echoes from F-layer in winter time by ionosonde in Kazan have shown frequent TID occurrence. Except traditionally observed by ionosonde the nose-shaped TIDs, corresponding to intensive irregularities, the focusing TIDs corresponding weak intensive irregularities are observed. In ionograms such focusing TID are visible as section of a F-layer traces with the raised amplitude and their revealing is possible only at such operating mode of the receiver when the reflected signals are not limited on amplitude. The given operating mode of the receiver together with speeded up mode of ionograms record ( $\mathrm{T} \leq 1$ min) allows to study distributions with amplitude of unities of percent and less, it means that the method of vertical sounding of an ionosphere has not settled still the opportunities in a direction of increase of resolution.

The up-to-date forms of data presentation and their processing allow reducing manual works at processing of such lot of data which arising at such frequent registration of ionograms. The three-dimensional array of AFC of F-layer traces considerably helps in automatic separation of TID in ionograms and ATF-plots helps for visual control of operation. Quality of ATF-plots is considerably refined at removal of noise with ionograms by means of a wavelet-filtration.

Such processing has allowed to determine that intervals between next TID are in a range of 4-20 min, with medial value of $10 \mathrm{~min}$. 


\section{References}

1. E.L. Afraimovich and N.P. Perevalova, GPS-monitoring of the Earth's upper atmosphere, Irkutsk, SC RRS SB RAMS, 2006, 480 p. (in russian).

2. J.W. MacDougall, G. Li and P.T. Jayachandran, "Traveling ionospheric disturbances near London, Canada," J. Atmos. and Solar-Terr. Phys., 71, 2009. pp. 2077-2084.

3. H.O. Booker, "The role of acoustic gravity waves in the generation of spread-F and ionospheric scintillation," J. Atmos. Terr. Phys., 41, 1979, pp. 501-515.

4. E.L. Afraimovich, The interference methods of radiosounding of an ionosphere, Moscow, Nauka, 1982 , 198p. (in russian).

5. Wave disturbances in an ionosphere / in T.B.Omarov (eds), Alma-Ata, Nauka, 1975, 179p. (in russian).

6. B.V. Troitskiy, The radiosounding signal response on ionospheric inhomogeneity, Alma-Ata, Nauka, 1983. 164p. (in russian).

7. A.D. Akchurin and V.V. Bochkarev, "Improved precision of virtual height measurements with coherent radio pulse sounding based on the maximum likelihood method," Adv. Space Res., 43, 2009, pp.1595-1602.

8. R.J. Lobb and J.E. Titheridge, "The effects of travelling ionospheric disturbances on ionograms," J. Atmos. and Terr. Phys. 39, 1977, pp.129-138. 